\title{
A comprehensive investigation of variants in genes encoding adiponectin (ADIPOQ) and its receptors (ADIPOR1/R2), and their association with serum adiponectin, type 2 diabetes, insulin resistance and the metabolic syndrome
}

\author{
Kirsten E Peters ${ }^{1}$, John Beilby ${ }^{2,3}$, Gemma Cadby ${ }^{4}$, Nicole M Warrington ${ }^{5}$, David G Bruce ${ }^{1}$, Wendy A Davis ${ }^{1}$, \\ Timothy ME Davis ${ }^{1}$, Steven Wiltshire ${ }^{6}$, Matthew Knuiman${ }^{7}$, Brendan M McQuillan, Lyle J Palmer ${ }^{9}$, \\ Peter $L$ Thompson ${ }^{8}$ and Joseph Hung ${ }^{8^{*}}$
}

\begin{abstract}
Background: Low levels of serum adiponectin have been linked to central obesity, insulin resistance, metabolic syndrome, and type 2 diabetes. Variants in $A D I P O Q$, the gene encoding adiponectin, have been shown to influence serum adiponectin concentration, and along with variants in the adiponectin receptors (ADIPOR1 and ADIPOR2) have been implicated in metabolic syndrome and type 2 diabetes. This study aimed to comprehensively investigate the association of common variants in ADIPOQ, ADIPOR1 and ADIPOR2 with serum adiponectin and insulin resistance syndromes in a large cohort of European-Australian individuals.

Methods: Sixty-four tagging single nucleotide polymorphisms in ADIPOQ, ADIPOR1 and ADIPOR2 were genotyped in two general population cohorts consisting of 2,355 subjects, and one cohort of 967 subjects with type 2 diabetes. The association of tagSNPs with outcomes were evaluated using linear or logistic modelling. Meta-analysis of the three cohorts was performed by random-effects modelling.

Results: Meta-analysis revealed nine genotyped tagSNPs in ADIPOQ significantly associated with serum adiponectin across all cohorts after adjustment for age, gender and BMl, including rs10937273, rs12637534, rs 1648707, rs16861209, rs822395, rs17366568, rs3774261, rs6444175 and rs17373414. The results of haplotype-based analyses were also consistent. Overall, the variants in the ADIPOQ gene explained $<5 \%$ of the variance in serum adiponectin concentration. None of the ADIPOR1/R2 tagSNPs were associated with serum adiponectin. There was no association between any of the genetic variants and insulin resistance or metabolic syndrome. A multi-SNP genotypic risk score for ADIPOQ alleles revealed an association with 3 independent SNPs, rs12637534, rs16861209, rs 17366568 and type 2 diabetes after adjusting for adiponectin levels ( $\mathrm{OR}=0.86,95 \% \mathrm{Cl}=(0.75,0.99), \mathrm{P}=0.0134)$.
\end{abstract}

Conclusions: Genetic variation in $A D I P O Q$, but not its receptors, was associated with altered serum adiponectin. However, genetic variation in ADIPOQ and its receptors does not appear to contribute to the risk of insulin resistance or metabolic syndrome but did for type 2 diabetes in a European-Australian population.

Keywords: Adiponectin, ADIPOQ, ADIPOR, Type 2 diabetes, Insulin resistance and Metabolic syndrome

\footnotetext{
*Correspondence: joe.hung@uwa.edu.au

${ }^{8}$ School of Medicine and Pharmacology, Sir Charles Gairdner Hospital Unit,

University of Western Australia, Nedlands, Western Australia, Australia

Full list of author information is available at the end of the article
} 


\section{Background}

Adiponectin is the most abundant adipose tissue-derived cytokine. It has anti-inflammatory, anti-diabetic and antiatherogenic properties, and low circulating levels are associated with central obesity, insulin resistance, metabolic syndrome (MetS), and type 2 diabetes (T2D) [1].

Serum adiponectin concentrations are highly heritable, and a number of genome-wide association studies (GWAS) have identified $A D I P O Q$, the gene encoding adiponectin, as the main locus contributing to variations in serum levels in European and Asian populations [2-5]. Cross-sectional studies in healthy and diabetic populations have provided further evidence for the association of single nucleotide polymorphisms (SNPs) in ADIPOQ with serum adiponectin concentrations [6-10]. Several studies have linked $A D I P O Q$ variants to $\mathrm{T} 2 \mathrm{D}$ and MetS, although the results to date have been discordant and not replicated across whole populations [5,8-12]. Nevertheless, examining the genes which affect serum adiponectin levels may help to confirm adiponectin as a cause or consequence of MetS and T2D using a Mendelian randomisation approach [13].

Adiponectin cellular signalling is mediated by two adiponectin receptors. The genes for these (ADIPOR1 and $A D I P O R 2)$, although generally not associated with serum adiponectin, have themselves been implicated in insulin resistance and T2D risk in genetic association studies, but also with inconsistent results [14-18].

In light of this background, the aims of the present study were i) to examine the evidence for association between total serum adiponectin levels and variants in $A D I P O Q$ and $A D I P O R 1 / R 2$ using a comprehensive tagSNP approach and haplotype-based analysis, ii) to assess associations between SNPs in these genes and total serum adiponectin concentrations in large, well-characterised and independent Western Australian cohorts (two general population and one adult T2D cohort), and iii) to investigate the relationship between gene variants found to be associated with adiponectin levels and relevant clinical outcomes including MetS and T2D.

\section{Methods}

\section{Subjects}

This study examined subjects from three Western Australian cohorts: the Busselton Population Health Survey (BHS) [19], the Carotid Ultrasound Disease Assessment Study (CUDAS) [20] and the Fremantle Diabetes Study (FDS) [21].

The BHS and CUDAS cohorts are representative of the general population and do not contain individuals with T2D, while FDS contains individuals with T2D who are representative of people with diabetes in an urban Australian setting. These cohorts are predominantly European-Australian. The present study was restricted to individuals who had both serum adiponectin and genotyping data available.
The subset of the BHS cohort with available data consisted of 1,322 unrelated adult individuals who were recruited as part of a larger cross-sectional community study in 1994/1995 [22]. The CUDAS cohort comprised 1,033 individuals selected from a random electoral survey from the Perth metropolitan area [20]. The FDS cohort of 967 diabetic individuals was recruited from a longitudinal observational study [21]. All study participants gave written informed consent, and the study protocol was approved by the University of Western Australia Human Research Ethics Committee, the Busselton Population Medical Research Foundation, and the Human Rights Committee at Fremantle Hospital.

\section{Biochemical and anthropomorphic analyses}

A fasting blood sample was obtained from each subject. Total serum adiponectin levels were measured by a commercially available quantitative sandwich enzyme immunoassay technique (R\&D Systems Inc., Minneapolis, Minnesota, USA). The intra-assay coefficients of variation ranged from 2.1 to $4.3 \%$ and the inter-assay coefficients of variation ranged from 5.4 to $9.6 \%$. Plasma glucose, insulin, total cholesterol, LDL- and HDL-cholesterol, and triglycerides were determined by standard methods [19-21]. Insulin resistance (IR) was determined using the homeostatic model of assessment scores (lnHOMA-IR) [23]. Anthropometric measurements and resting blood pressure were taken according to standard clinical procedures. Body mass index (BMI) was calculated as weight $(\mathrm{kg}) /$ height $(\mathrm{m})^{2}$. Metabolic syndrome (MetS) was defined according to the recently updated National Cholesterol Education Program (NCEP) criteria [24]. Individuals with three or more of the following criteria were classified as having MetS: increased waist circumference ( $\geq 102 \mathrm{~cm}$ in men, $\geq 88 \mathrm{~cm}$ in women), hypertriglyceridaemia $\geq 1.70 \mathrm{mmol} / \mathrm{L}(\geq 150 \mathrm{mg} / \mathrm{dL})$, low HDL-cholesterol $<1.00 \mathrm{mmol} / \mathrm{L}$ in men and $<1.3 \mathrm{mmol} / \mathrm{L}$ in women $(<40 \mathrm{mg} / \mathrm{dL}$ and $<50 \mathrm{mg} / \mathrm{dL})$, high blood pressure (systolic $\geq 130$ and/or diastolic $\geq 85 \mathrm{~mm} \mathrm{Hg}$, or current use of antihypertensive therapy) and high fasting glucose $\geq 5.6 \mathrm{mmol} / \mathrm{L}$ ( $\geq 100 \mathrm{mg} / \mathrm{dL}$ ). Data for clinical and demographic characteristics (Table 1) are given as proportions, mean $\pm \mathrm{SD}$, or geometric mean (SD range).

\section{Selection and genotyping of tSNPs}

A tagSNP (tSNP) approach $\left(r^{2} \geq 0.80\right.$, minor allele frequency $\geq 5 \%$ ) was used to explore the genetic variation in $A D I P O Q$, ADIPOR1, and ADIPOR2. tSNPs were selected using Haploview to represent the common genetic variation of each gene, including an additional $10 \mathrm{~kb}$ upstream and downstream, as well as variants previously reported in the literature to be associated with serum adiponectin levels. Sixty-four tSNPs in ADIPOQ (8), ADIPOR1 and ADIPOR2 $(15,16)$ were selected and genotyped based on this approach (Additional file 1). Genotyping was performed on 
Table 1 Baseline clinical, metabolic, and demographic characteristics of each cohort by gender

\begin{tabular}{|c|c|c|c|c|c|c|}
\hline & \multicolumn{2}{|c|}{ BHS } & \multicolumn{2}{|c|}{ CUDAS } & \multicolumn{2}{|c|}{ FDS } \\
\hline & Female $(n=736)$ & Male $(n=586)$ & Female $(n=521)$ & Male $(n=512)$ & Female $(n=489)$ & Male $(n=478)$ \\
\hline Adiponectin (mg/L) & $14.2(8.1-24.8)$ & $7.9(4.2-15.0)$ & $13.9(8.1-23.8)$ & $7.9(4.5-14.0)$ & $8.6(4.4-16.6)$ & $5.9(3.0-11.4)$ \\
\hline Age (years) & $51.3 \pm 17.8$ & $51.5 \pm 17.7$ & $52.8 \pm 12.7$ & $53.2 \pm 12.8$ & $64.6 \pm 11.4$ & $64.4 \pm 10.3$ \\
\hline $\mathrm{SBP}(\mathrm{mm} \mathrm{Hg})$ & $122 \pm 20$ & $126 \pm 16$ & $126 \pm 20$ & $129 \pm 16$ & $150 \pm 23$ & $151 \pm 23$ \\
\hline $\mathrm{DBP}(\mathrm{mm} \mathrm{Hg})$ & $73 \pm 10$ & $77 \pm 10$ & $78 \pm 11$ & $82 \pm 9$ & $79 \pm 11$ & $83 \pm 11$ \\
\hline Fasting Glucose (mmol/L) & $4.8 \pm 0.5$ & $4.9 \pm 0.5$ & $5.3 \pm 0.7$ & $5.3 \pm 0.8$ & $8.7(6.3-12.0)$ & $8.6(6.2-11.8)$ \\
\hline Fasting Insulin $(I \cup / L)^{*}$ & $41.6(24.6-70.3)$ & $44.6(24.6-80.8)$ & $33.6(18.4-61.4)$ & $36.1(19.1-68.5)$ & $80.7(44.2-147.2)$ & $68.0(34.6-133.4)$ \\
\hline InHOMA-IR (insulin resistance) & $0.9(0.5-1.5)$ & $0.9(0.5-1.7)$ & $0.6(0.3-1.2)$ & $0.7(0.4-1.3)$ & $1.7(0.9-3.2)$ & $1.4(0.7-2.8)$ \\
\hline Total cholesterol (mmol/L) & $5.6 \pm 1.2$ & $5.5 \pm 1.0$ & $5.6 \pm 1.0$ & $5.5 \pm 1.0$ & $5.7 \pm 1.1$ & $5.2 \pm 1.1$ \\
\hline LDL-cholesterol (mmol/L) & $3.5 \pm 1.0$ & $3.7 \pm 0.9$ & $3.6 \pm 0.9$ & $3.7 \pm 0.9$ & $3.6 \pm 1.0$ & $3.3 \pm 0.8$ \\
\hline HDL-cholesterol (mmol/L) & $1.51(1.17-1.95)$ & $1.18(0.91-1.54)$ & $1.47(1.14-1.88)$ & $1.14(0.89-1.47)$ & $1.09(0.82-1.46)$ & $0.93(0.68-1.26)$ \\
\hline Serum triglycerides (mmol/L) & $1.0(0.6-1.7)$ & $1.2(0.7-2.2)$ & $1.0(0.6-1.6)$ & $1.2(0.7-2.1)$ & $1.9(1.2-3.0)$ & $1.9(1.0-3.4)$ \\
\hline Body Mass Index (BMI) $\left(\mathrm{kg} / \mathrm{m}^{2}\right)$ & $25.1 \pm 4.4$ & $26.3 \pm 3.5$ & $25.3 \pm 4.4$ & $26.6 \pm 3.5$ & $30.2 \pm 5.9$ & $28.9 \pm 4.5$ \\
\hline Waist circumference $(\mathrm{cm})$ & $79.7 \pm 11.0$ & $92.9 \pm 10.2$ & $76.9 \pm 10.4$ & $92.0 \pm 9.2$ & $96.4 \pm 12.6$ & $103.4 \pm 11.2$ \\
\hline Waist-Hip ratio & $0.79 \pm 0.06$ & $0.93 \pm 0.06$ & $0.76 \pm 0.06$ & $0.90 \pm 0.05$ & $0.86 \pm 0.06$ & $0.96 \pm 0.06$ \\
\hline Antihypertensive tx (\%) & 19.2 & 16.1 & 13.8 & 13.9 & 56.4 & 45.4 \\
\hline Smoking (never/ex/current) (\%) & $60.2 / 28.9 / 10.9$ & $47.6 / 37.1 / 15.2$ & $62.8 / 24.2 / 13.1$ & $39.1 / 43.6 / 17.4$ & $64.4 / 23.6 / 12.0$ & 24.9/57.4/17.6 \\
\hline NCEP_Hypertension (\%) & 40.0 & 46.2 & 47.0 & 54.7 & 92.8 & 91.0 \\
\hline NCEP_Hyperglycaemia (\%) & 7.1 & 10.0 & 33.9 & 40.2 & 94.3 & 93.5 \\
\hline NCEP_Hypertriglyceridemia (\%) & 17.4 & 28.9 & 14.8 & 30.7 & 59.0 & 58.7 \\
\hline NCEP_LOWHDL (\%) & 27.3 & 26.8 & 30.1 & 29.3 & 73.1 & 60.0 \\
\hline NCEP_Obese (\%) & 20.7 & 18.1 & 16.3 & 14.8 & 74.1 & 55.0 \\
\hline \multicolumn{7}{|l|}{ NCEP_MetSScore ${ }^{\dagger}(\%)$} \\
\hline 0 & 39.8 & 31.7 & 27.2 & 18.6 & 0.4 & 0.2 \\
\hline 1 & 29.9 & 32.3 & 29.5 & 27.1 & 1.0 & 2.0 \\
\hline 2 & 16.6 & 18.3 & 26.4 & 29.6 & 8.5 & 15.8 \\
\hline $3+$ & 13.7 & 17.7 & 17.0 & 24.7 & 90.0 & 82.0 \\
\hline
\end{tabular}

* insulin in non-insulin users ${ }^{\dagger}$ NCEP_MetSScore is the number of MetS components.

The data for continuous variables is mean \pm standard deviation, or geometic mean (standard deviation range). The data for categorical variables is shown as proportions.

an Illumina BeadStation using the GoldenGate technology. DNA samples from CEPH trios (obtained from the Coriell Cell Repository) served as internal controls for quality of clustering and reproducibility. A random (10\%) sample was analysed in duplicate, with reproducibility found to be $100 \%$ for the Busselton population and $99.9 \%$ for both CUDAS and FDS populations. Individual SNP concordance rates were between 99.3 and 100\%. Deviations from HardyWeinberg equilibrium (HWE) $(\mathrm{P}<0.05)$ were examined for each tSNP using Fisher's exact test, and minor allele frequencies determined for each cohort.

\section{Analysis of association between tSNPs and adiponectin levels}

Adiponectin levels were normalised using a natural logarithm transformation prior to analysis. Associations between transformed values and genotypes at each tSNP were examined using a generalised linear model approach implemented in SimHap [25]. Each polymorphism was modelled as a genotypic (codominant) genetic effect, accommodating the effects of age, gender, and BMI as significant covariates. We determined marginal geometric mean values of adiponectin according to genotype at each tSNP using SimHap. We corrected for the multiple testing inherent in this study using the false discovery rate method, and provide q-values [26] (Additional file 2). Statistical significance was defined at $\alpha<0.05$.

We used Haploview [27] to determine the linkage disequilibrium (LD) between the selected tSNPs and characterise the haplotype block structure. Haplotypes were inferred for individuals with ambiguous phase and haplotype frequencies were estimated using an expectation- 
maximization algorithm as implemented in SimHap. Haplotypes were recorded as independent factors into three classes $(0,1$, or 2$)$, representing the number of copies of each haplotype in an individual's diplotype. The effect of each individual haplotype was calculated relative to not having that haplotype. To investigate associations with serum adiponectin, common haplotypes (frequency $>5 \%$ ) were examined using a codominant model adjusted for age, gender and BMI (Additional file 3).

Meta-analysis of the ADIPOQ polymorphisms against total serum adiponectin in the three cohorts was carried out using the rmeta package in $\mathrm{R}$ version 2.8.0. All three cohorts were pooled after checking for genetic heterogeneity for each tSNP. Random-effects modelling were used for the meta-analysis as we assumed that the cohorts would be heterogeneous.

\section{Analysis of association between tSNPs and T2D, InHOMA- IR and MetS}

Associations between tSNPs and T2D, log transformed HOMA-IR (InHOMA-IR) and MetS were evaluated in SimHap using a codominant model corrected for age, gender and BMI. For T2D, the FDS cohort was compared to control individuals without a history of diabetes from the combined BHS and CUDAS cohorts. LnHOMA-IR was modelled as a quantitative outcome, while MetS was analysed as an ordinal outcome in the BHS and CUDAS cohorts for the number of MetS criteria met. In addition to investigating the tSNPs independently, an allele score was created by summing the number of risk alleles an individual had in those tSNPs that were shown to be associated with adiponectin levels in the meta-analysis.

\section{Power analysis}

A power calculation, performed in the Quanto software [28], indicated that this study has at least $90 \%$ power at an alpha level of $\mathrm{p}=0.05$ to detect an odds ratio for diabetes (population risk of 10\%) of 1.45 for a minor allele frequency of 0.05 and an odds ratio of 1.2 for a minor allele frequency of 0.45 , under an additive genetic model.

\section{Results}

\section{Sample characteristics}

A total of 3,322 subjects from the three cohorts were included (Table 1). As expected, the two general populationbased cohorts differed significantly to the diabetic cohort in cardiometabolic risk characteristics, reflecting the different recruitment criteria. The BHS and CUDAS populations were similar with regards serum adiponectin concentrations, age and BMI. Compared with the BHS and CUDAS cohorts, the FDS population was older, had lower serum adiponectin concentrations, and had higher BMI and systolic blood pressure. In all three cohorts, females had higher serum adiponectin concentrations than males.

\section{Genotype distribution and linkage disequilibrium}

Of the sixty-four genotyped tSNPs, five failed and six were monomorphic, leaving fifty-three that were included in the association analyses (21 in ADIPOQ, 13 in ADIPOR1, and 19 in $A D I P O R 2)$. Details of these tSNPs including genotype and allele frequencies are reported in Additional file 1. Allele frequencies for all tSNPs were similar to those reported in HapMap (http://hapmap.ncbi.nlm.nih.gov/). The genotype distribution of each tSNP was consistent with HWE in all populations, with the following exceptions: two tSNPs in BHS (ADIPOR1 rs75114693 and ADIPOR1 rs6666089) and one tSNP in CUDAS (ADIPOR1 rs6666089) (all $P<0.05$ ).

Analysis of pairwise LD in ADIPOQ showed three LD blocks (Figure 1), which were similar between all three cohorts. Haplotypes were analysed within each block for association with the outcomes of interest. A strong correlation ( ${ }^{\prime}>0.90$ and $r^{2}>0.8$ ) was found between tSNPs rs6810075 and rs1648707, rs864265 and rs860291, rs822387 and rs16861209, rs822391 and rs822396, and rs2241766 and rs1063537 within each cohort. Statistical analysis showed that the effects of each of these tSNP pairs on adiponectin levels were identical, and for this reason we chose to report on only one tSNP from each pair: rs1648707, rs860291, rs16861209, rs822396 and rs1063537. There was no interaction between gender and any of the genetic variants, so males and females were combined for all subsequent analyses.

\section{Association of single tSNPs with serum adiponectin}

Age, gender and BMI accounted for $35.5 \%$ of the variation in serum adiponectin in the BHS cohort, $33.2 \%$ in CUDAS, and $22.0 \%$ in FDS. After adjustment for these covariates and, under a codominant model, eight tSNPs in $A D I P O Q$ were significantly associated with serum adiponectin in the BHS cohort: rs12637534, rs1648707, rs16861209, rs17366568, rs3774261, rs1063537, rs6444175 and rs17373414 (all $\mathrm{q}<0.05$ ). Of these, four were significant in CUDAS (rs1648707, rs16861209, rs3774261, and rs6444175), and two in FDS (rs16861209 and rs17366568). Full results (for these eight SNPs) for each cohort, including the data for tSNPs in LD, are shown in Additional file 2. None of the tSNPs in $A D I P O R 1 / R 2$ were associated with serum adiponectin in any of the cohorts.

After adjustment for age, gender and BMI, tSNPs rs12637534, rs16861209 and rs17366568 accounted for 4.8\% of the variance in serum adiponectin in BHS, rs1648707 and rs16861209 explained 2.9\% of the variance in CUDAS, and rs16861209 and rs17366568 accounted for 1.7\% in FDS. The minor allele at rs16861209 (A allele) was consistently associated with increased serum adiponectin across all three cohorts, specifically $3.7 \mathrm{mg} / \mathrm{L}$ in BHS, $2.7 \mathrm{mg} / \mathrm{L}$ in CUDAS and $1.3 \mathrm{mg} / \mathrm{L}$ in FDS (Additional file 2).

Meta-analysis of all $A D I P O Q$ tSNPs in our three cohorts is shown in Table 2. tSNPs in LD gave identical 


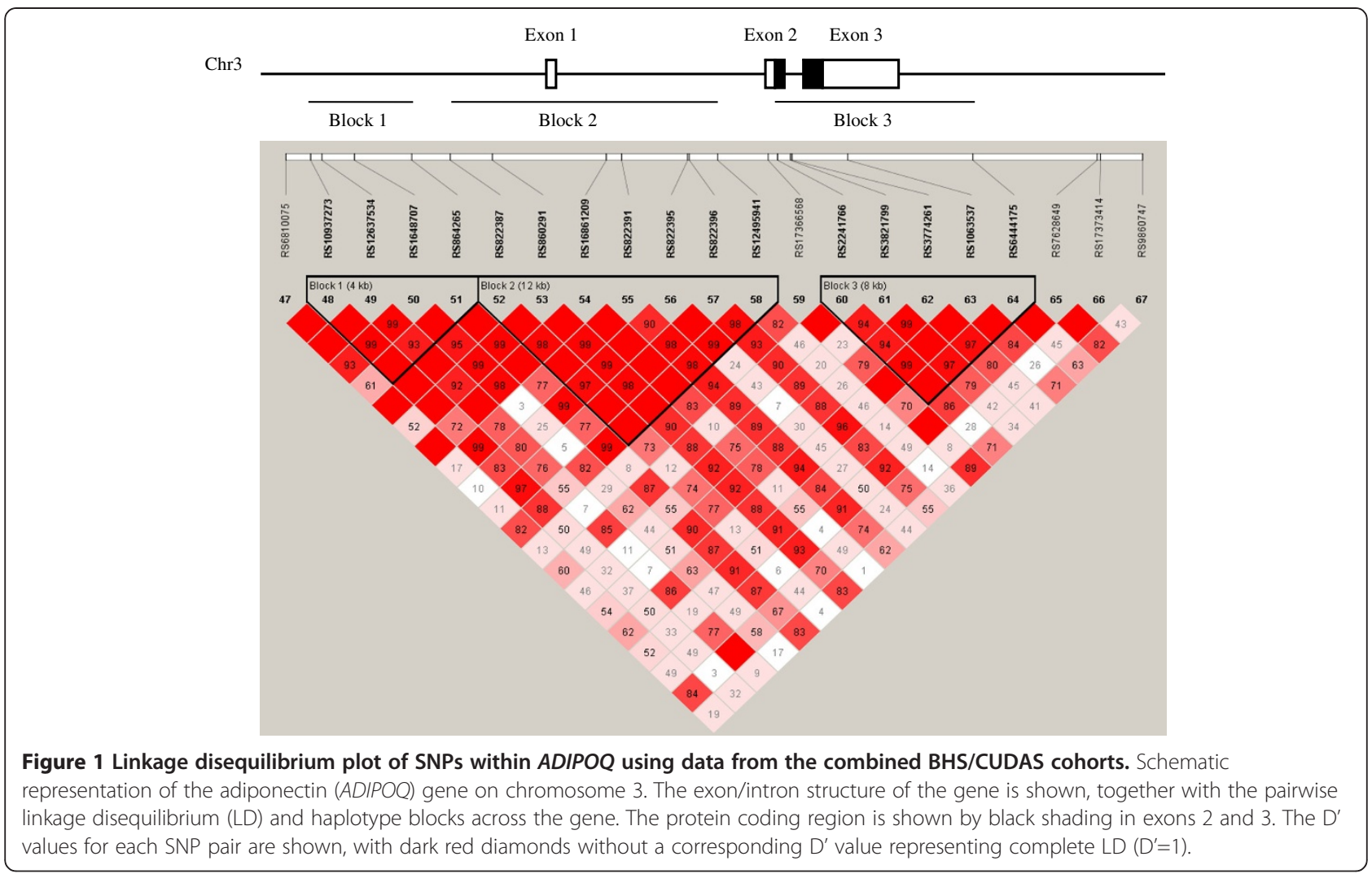

results so only data for one tSNP from each pair are presented. As FDS displayed different mean levels of adiponectin and other quantative traits, a second metaanalysis was conducted without the FDS study and similar results were found (data not shown).

The meta-analysis showed a consistent, homogeneous effect of seven of the eight tSNPs associated with serum adiponectin in the BHS cohort $(\mathrm{q}<0.05)$. tSNP rs1063537 (and rs2241766 via strong LD) showed significant evidence of heterogeneity $(P=0.001)$, indicating differences between the cohorts at this marker. Two additional tSNPs (rs10937273 and rs822395) were also associated with serum adiponectin in the meta-analysis of the three combined cohorts. Carriage of the minor alleles at rs12637534, rs1648707 and rs17366568 was associated with lower serum adiponectin levels $\left(P<1 \times 10^{-5}\right)$, while the minor alleles at rs10937273, rs16861209, rs822395, rs3774261, rs6444175 and rs17373414 was associated with higher serum adiponectin levels $(P \leq 0.02)$.

To determine which of the 9 SNPs identified in the meta-analysis independently contribute to adiponectin variation, we conducted a conditional regression analysis in all three cohorts; this analysis showed that 3 of the top 9 SNPs were independently associated with adiponectin levels in the BHS, including rs12637534, rs16861209 and rs17366568. Similar results were found in the other two studies, where both rs16861209 and rs17366568 remained significant in FDS, while rs16861209, rs17366568 and rs1648707 were significant in CUDAS.

\section{Associations of haplotypes with serum adiponectin}

Associations between the most frequent haplotypes and serum adiponectin were analysed after correcting for age, gender and BMI in each cohort (data not shown). All cohorts were combined and meta-analysis performed on these haplotypes using random effects modelling (Table 3). The haplotype-based associations were consistent with the results from the single tSNP analyses.

In block 1, five common haplotypes were observed that significantly affected serum adiponectin. Haplotypes carrying the major allele at rs12637534 and rs1648707 (A-A) were found to increase serum adiponectin (all $P \leq 9.20 \times 10^{-3}$ ), with the exception of the GAAT haplotype where one copy was associated with decreased serum adiponectin concentrations $(P=0.03)$. The opposite was true for carriage of the minor allele at each of these tSNPs, with one copy of GGCG associated with a significantly decreased serum adiponectin $\left(P=8.22 \times 10^{-7}\right)$. One haplotype, GACG, carrying the major allele at rs12637534 (A) and minor allele at rs1648707 (C), was also associated with a decreased serum adiponectin, suggesting that the effect of rs1648707 may be stronger than rs12637534. This seems likely given the lower minor allele frequency of rs12637534 compared to rs1648707 (8\% vs 34\%). 
Table 2 Meta-analysis of the association between ADIPOQ SNPs and serum adiponectin in our combined cohort of 3,431 individuals

\begin{tabular}{|c|c|c|c|c|}
\hline SNP & Number of individuals & $\beta(95 \% \mathrm{Cl})$ & P-value & P-value (heterogeneity) \\
\hline \multicolumn{5}{|c|}{ rs10937273 } \\
\hline GA & 1576 & $0.0004(-0.041,0.041)$ & 0.98 & 0.63 \\
\hline AA & 577 & $0.081(0.027,0.134)$ & $3.30 \mathrm{E}-03$ & 0.83 \\
\hline \multicolumn{5}{|c|}{ rs12637534 } \\
\hline$A G$ & 481 & $-0.147(-0.209,-0.085)$ & 3.39E-06 & 0.26 \\
\hline GG & 18 & $0.076(-0.171,0.323)$ & 0.55 & 0.63 \\
\hline \multicolumn{5}{|c|}{ rs1648707 } \\
\hline$A C$ & 1473 & $-0.090(-0.129,-0.050)$ & 8.06E-06 & 0.44 \\
\hline CC & 392 & $-0.192(-0.252,-0.131)$ & $5.41 \mathrm{E}-10$ & 0.80 \\
\hline \multicolumn{5}{|c|}{ rs860291 } \\
\hline CT & 767 & $0.028(-0.017,0.072)$ & 0.22 & 0.61 \\
\hline$\pi$ & 50 & $0.089(-0.064,0.243)$ & 0.25 & 0.97 \\
\hline \multicolumn{5}{|c|}{ rs16861209 } \\
\hline CA & 530 & $0.246(0.179,0.313)$ & $8.14 \mathrm{E}-13$ & 0.17 \\
\hline AA & 27 & $0.337(0.129,0.545)$ & $1.50 \mathrm{E}-03$ & 0.61 \\
\hline \multicolumn{5}{|c|}{ rs822395 } \\
\hline$A C$ & 1465 & $0.052(0.007,0.098)$ & 0.02 & 0.28 \\
\hline CC & 404 & $0.069(0.009,0.129)$ & 0.03 & 0.88 \\
\hline \multicolumn{5}{|c|}{ rs822396 } \\
\hline$A G$ & 998 & $0.002(-0.039,0.043)$ & 0.92 & 0.90 \\
\hline GG & 129 & $0.010(-0.087,0.108)$ & 0.84 & 0.49 \\
\hline \multicolumn{5}{|c|}{ rs12495941 } \\
\hline GT & 1500 & $0.006(-0.034,0.046)$ & 0.77 & 0.41 \\
\hline$\pi$ & 386 & $0.056(-0.035,0.148)$ & 0.23 & 0.11 \\
\hline \multicolumn{5}{|c|}{ rs17366568 } \\
\hline GA & 691 & $-0.146(-0.205,-0.087)$ & $1.22 \mathrm{E}-06$ & 0.19 \\
\hline AA & 41 & $-0.370(-0.543,-0.197)$ & $2.72 \mathrm{E}-05$ & 0.34 \\
\hline \multicolumn{5}{|c|}{ rs3821799 } \\
\hline CT & 1573 & $0.024(-0.034,0.082)$ & 0.42 & 0.16 \\
\hline$\pi$ & 685 & $0.051(-0.002,0.104)$ & 0.06 & 0.52 \\
\hline \multicolumn{5}{|c|}{ rs3774261 } \\
\hline GA & 1502 & $0.060(0.019,0.010)$ & $3.80 \mathrm{E}-03$ & 0.63 \\
\hline AA & 517 & $0.176(0.120,0.231)$ & $4.98 \mathrm{E}-10$ & 0.60 \\
\hline \multicolumn{5}{|c|}{ rs1063537 } \\
\hline CT & 659 & $0.026(-0.045,0.096)$ & 0.48 & 0.11 \\
\hline$\pi$ & 47 & $0.233(-0.203,0.669)$ & 0.29 & 0.001 \\
\hline \multicolumn{5}{|c|}{ rs6444175 } \\
\hline GA & 1300 & $0.067(0.028,0.106)$ & $7.00 \mathrm{E}-04$ & 0.45 \\
\hline AA & 229 & $0.180(0.105,0.255)$ & 2.40E-06 & 0.74 \\
\hline \multicolumn{5}{|c|}{ rs7628649 } \\
\hline $\mathrm{CT}$ & 656 & $0.031(-0.015,0.077)$ & 0.19 & 0.51 \\
\hline$\pi$ & 47 & $0.188(-0.082,0.458)$ & 0.17 & 0.05 \\
\hline
\end{tabular}


Table 2 Meta-analysis of the association between ADIPOQ SNPs and serum adiponectin in our combined cohort of 3,431 individuals (Continued)

\begin{tabular}{lcccc}
\hline rs17373414 & & & & \\
CT & 654 & $0.116(0.069,0.163)$ & $1.64 \mathrm{E}-06$ & 0.35 \\
$\pi$ & 35 & $-0.004(-0.186,0.179)$ & 0.97 & 0.39 \\
rs9860747 & & & & 0.67 \\
$C T$ & 871 & $0.018(-0.024,0.061)$ & 0.40 & 0.43 \\
$T$ & 90 & $0.073(-0.042,0.187)$ & 0.21 & \\
\hline
\end{tabular}

In block 2, the CCATCAG haplotype (frequency 8\%) carrying the minor alleles at rs16861209 (A) and rs822395 (C) (both associated with a higher serum adiponectin) was found to be significantly associated with serum adiponectin $\left(P=5.11 \times 10^{-15}\right)$. This haplotype was found to be significant in each cohort and explained $2.3 \%$ of the variation in levels in BHS, $2.0 \%$ in CUDAS and $0.7 \%$ in FDS. Conversely, three haplotypes carrying the major allele at rs16861209 (associated with a lower serum adiponectin) were found to be significantly associated with a decreased serum adiponectin (all $P \leq 3.11 \times 10^{-2}$ ).

Two haplotypes in block 3 were significantly associated with serum adiponectin in the meta-analysis, and in each individual cohort. The TTACA haplotype (frequency $\sim 25 \%$ ) was associated with increased levels and TTGCG (frequency $\sim 6 \%$ ) with decreased levels $\left(P \leq 4 \times 10^{-4}\right.$ and $P \leq 2.87 \times 10^{-12}$, respectively). The TTACA haplotype carries the minor allele (A allele) at both rs3774261 and rs6444175 that was shown in the single tSNP analyses to be associated with increased serum adiponectin, while TTGCG carries the major allele at each of these tSNPs and has the opposite effect on levels, again showing consistency between single tSNP and haplotype analysis. This haplotype accounted for $2.3 \%$ of the variation in levels in BHS, $0.7 \%$ in CUDAS and $0.6 \%$ in FDS. The more common TTACA haplotype only accounted for between 0.3 and $0.7 \%$ of the variation in levels across all three cohorts.

In all haplotype blocks, the single SNPs tagging the block accounted for the same, or slightly more of the variation in adiponectin levels than the haplotype block itself (difference of between $0-0.8 \%$ between the tSNPs and the haplotypes).

\section{Association of tSNPs with T2D, InHOMA-IR and MetS}

No significant associations were detected between genetic variants in $A D I P O Q$, or $A D I P O R 1 / R 2$, and InHOMA-IR or MetS in any cohort after correction for age, gender and BMI. We also found no association between the ADIPOQ and $A D I P O R 1 / R 2$ genetic variants and T2D in the case: control analysis (results not shown).

There was a significant association between the allele score of the top nine SNPs and T2D in the case-control study without adjusting for adiponectin levels $(\mathrm{OR}=0.94,95 \% \mathrm{CI}=$ (0.91,0.98), $\mathrm{P}=0.0015)$ and this association strengthened after adjusting for adiponectin levels $(\mathrm{OR}=0.92,95 \% \mathrm{CI}=(0.89$, 0.96), $\left.\mathrm{P}=2.43 \times 10^{-5}\right)$. The allele score of the 3 independent SNPs; rs12637534, rs16861209, rs17366568, were significantly associated with T2D only after adjusting for adiponectin levels $(\mathrm{OR}=0.86,95 \% \mathrm{CI}=(0.75,0.99), \mathrm{P}=0.0134)$.

\section{Discussion}

The present study reports associations between total serum adiponectin concentrations and a range of genetic variations and haplotypes in the adiponectin gene $(A D I P O Q)$ and genes for adiponectin receptors 1 and 2 $(A D I P O R 1 / R 2)$ in three independent European-Australian population-based cohorts. Several genetic variants in $A D I P O Q$, but not $A D I P O R 1 / R 2$, were associated with serum adiponectin. These associations were consistent across the three cohorts and showed similar results for tSNP and haplotype-based analysis. After adjusting for age, gender, and $\mathrm{BMI}$, variants in the $A D I P O Q$ gene accounted for only $3.9 \%$ of the variation in serum adiponectin in the combined general population cohorts and $1.7 \%$ in the diabetic sample. There was also no association between tSNPs or haplotypes of ADIPOQ, and ADIPOR1/ $R 2$ with T2D, insulin resistance assessed by lnHOMA-IR, or MetS.

This relatively large study confirms that ADIPOQ tSNPs are significantly associated with serum adiponectin. We found the minor alleles at rs12637534, rs1648707/ rs6810075 and rs17366568 to be associated with lower serum adiponectin concentration $\left(P<1 \times 10^{-5}\right)$, while the minor alleles at rs10937273, rs16861209/rs822387, rs822395, rs3774261, rs6444175 and rs17373414 were associated with higher levels $(P \leq 0.02)$. These associations and their direction are consistent with the hypothesis that circulating adiponectin levels are in part determined by genetic influences [3-7,9,10,29-31].

We failed to replicate previous findings in the literature for ADIPOQ SNPs rs2241766, rs1063537 and rs3821799 that have been reported in two large GWAS [3,31], but not supported by other studies $[4,8]$. In addition, there were three tSNPs (rs266729, rs62625753 and rs17366743) reported as associated with serum adiponectin that did 
Table 3 Meta-analysis of the association between ADIPOQ haplotypes and serum adiponectin in our combined cohort of 3,431 individuals

\begin{tabular}{|c|c|c|c|c|}
\hline Block & Haplotype and copy number & $\beta(95 \% \mathrm{Cl})$ & P-value & P-value (heterogeneity) \\
\hline \multirow[t]{15}{*}{1} & AAAG & & & \\
\hline & 1 & $0.001(-0.041,0.043)$ & 0.96 & 0.67 \\
\hline & 2 & $0.081(0.026,0.135)$ & $3.70 \mathrm{E}-03$ & 0.74 \\
\hline & GACG & & & \\
\hline & 1 & $-0.071(-0.110,-0.032)$ & 4.00E-04 & 0.79 \\
\hline & 2 & $-0.109(-0.183,-0.035)$ & $3.70 \mathrm{E}-03$ & 0.61 \\
\hline & GAAT & & & \\
\hline & 1 & $-0.047(-0.089,-0.005)$ & $3.00 E-02$ & 0.62 \\
\hline & 2 & $0.026(-0.099,0.152)$ & 0.68 & 0.85 \\
\hline & GAAG & & & \\
\hline & 1 & $0.219(0.142,0.296)$ & $2.55 \mathrm{E}-08$ & 0.11 \\
\hline & 2 & $0.280(0.070,0.490)$ & $9.20 \mathrm{E}-03$ & 0.89 \\
\hline & GGCG & & & \\
\hline & 1 & $-0.152(-0.231,-0.092)$ & $8.22 \mathrm{E}-07$ & 0.28 \\
\hline & 2 & $0.051(-0.188,0.291)$ & 0.67 & 0.60 \\
\hline \multirow[t]{18}{*}{2} & TCCTAAT & & & \\
\hline & 1 & $0.007(-0.036,0.049)$ & 0.76 & 0.34 \\
\hline & 2 & $0.052(-0.048,0.153)$ & 0.31 & 0.08 \\
\hline & TCCTAAG & & & \\
\hline & 1 & $-0.069(-0.108,0.030)$ & $6.00 E-04$ & 0.54 \\
\hline & 2 & $-0.115(-0.183,-0.046)$ & $1.00 \mathrm{E}-03$ & 0.64 \\
\hline & TTCCCGG & & & \\
\hline & 1 & $0.026(-0.021,0.073)$ & 0.28 & 0.73 \\
\hline & 2 & $0.122(-0.035,0.278)$ & 0.13 & 0.96 \\
\hline & CCATCAG & & & \\
\hline & 1 & $0.244(0.183,0.305)$ & $5.11 \mathrm{E}-15$ & 0.29 \\
\hline & 2 & $0.301(0.079,0.523)$ & 7.80E-03 & 0.88 \\
\hline & TCCTCAG & & & \\
\hline & 1 & $-0.156(-0.228,-0.084)$ & $2.42 \mathrm{E}-05$ & 0.22 \\
\hline & 2 & $-0.402(-0.066,-0.146)$ & $2.10 \mathrm{E}-03$ & 0.74 \\
\hline & TCCCCGG & & & \\
\hline & 1 & $-0.067(-0.129,-0.006)$ & $3.11 \mathrm{E}-02$ & 0.37 \\
\hline & 2 & $0.088(-0.217,0.393)$ & 0.57 & 0.45 \\
\hline \multirow[t]{9}{*}{3} & TCGCG & & & \\
\hline & 1 & $-0.030(-0.081,0.022)$ & 0.25 & 0.34 \\
\hline & 2 & $-0.044(-0.096,0.008)$ & 0.09 & 0.44 \\
\hline & TTACA & & & \\
\hline & 1 & $0.071(0.032,0.111)$ & 4.00E-04 & 0.57 \\
\hline & 2 & $0.190(0.114,0.267)$ & $1.01 \mathrm{E}-06$ & 0.88 \\
\hline & GTATG & & & \\
\hline & 1 & $0.022(-0.049,0.094)$ & 0.54 & 0.12 \\
\hline & 2 & $0.220(-0.207,0.647)$ & 0.31 & 0.001 \\
\hline
\end{tabular}


not show a significant association with adiponectin levels in the present study. LD data available from Heid et al. [3] show that $\mathrm{rs} 266729$ is correlated $\left(\mathrm{r}^{2}>0.60\right.$, $\left.\mathrm{D}^{\prime}>0.80\right)$ with tSNPs which were significant in our study (rs6810075, rs182052 and rs1648707).

The lack of replication of the association between adiponectin levels and SNPs previously identified in GWAS is intriguing. As several rare population-specific coding variants have been reported to be strongly associated with adiponectin level $[6,32,33]$ One possible explanation for the observed discrepancies may be due to synthetic associations i.e. the fact that population-specific rare variants are in partial linkage disequilibrium with common variants [34].

In the present study, the significantly associated tSNPs were located across all regions of the gene, including the promoter, exonic, intronic and 3 -untranslated regions. Interestingly, all of the tSNPs downstream of exon 2 were associated with an increased serum adiponectin.

We observed no association between ADIPOR1/R2 variants and serum adiponectin which is consistent with most other studies [14,35-37]. Recent genome-wide studies have identified variants in a third adiponectin receptor, T-cadherin, that affect circulating adiponectin $[2,4,38,39]$. T-cadherin, encoded by $C D H 13$, is a receptor for hexameric and high-molecular-weight adiponectin, but not for the trimeric or globular species as measured in the present study. Nevertheless, variants in $\mathrm{CDH} 13$ warrant further investigation.

Despite the fact that $A D I P O Q$ variants are associated with a modest amount of variance in total serum adiponectin $(<5 \%)$, interventions aimed at the encoded protein may affect secreted adiponectin to a much greater extent. Indeed, the thiazolidinediones have been shown to increase circulating adiponectin $2-2.5$ fold in patients with diabetes, although the exact mechanism of action remains unknown. Other population studies have reported similar quantitatively small effects of $A D I P O Q$ SNPs, and show that there are a large number of SNPs contributing independently to the variation $[3,7,40]$. A recent large multiethnic GWAS identified ten new loci, in addition to $A D I P O Q$ and $C D H 13$, that affect circulating adiponectin, but the combined multi-SNP genotypic risk score only explained $5 \%$ of the variance in serum adiponectin [31]. The high heritability estimates for adiponectin may be explained by as-yet undiscovered rare variants with strong effects, additional unknown common loci with small effects, epigenetic mechanisms, or by copy number variations (CNVs). ADIPOQ contains a high number of CNVs and the effect of these on circulating adiponectin is unknown. Complete sequencing of the $A D I P O Q$ locus has been performed in a large population where seven SNPs were shown to influence adiponectin levels but the authors found no evidence of an association between these SNPs and T2D in a diabetic case control study [33].

Recently, studies have identified rare population-specific mutations that account for $17 \%$ of the variance in serum adiponectin [32,41]. Further analysis of gene-gene and gene-environment interactions may explain the remaining heritability.

Despite putative effects of the adiponectin receptors on tissue adiponectin activity, we found no evidence that tSNPs in $A D I P O Q$, or $A D I P O R 1 / R 2$ were associated with either insulin resistance, or MetS. This supports the findings of two other studies [5,33]. However, we did find a significant association between the allele score of the top nine SNPs and T2D in the case-control study without adjusting for adiponectin levels. Multi-SNP genotypic risk score for adiponectin-decreasing alleles has been associated with BMI, WHR, fasting insulin, HOMA-IR, 2-hour post OGTT glucose, T2D, triglycerides and HDL-cholesterol [31]. In the current study a post-hoc analysis revealed that adjusting for adiponectin levels strengthened the association with 3 independent SNPs, rs12637534, rs16861209, rs17366568 and T2D. The strengthening of this association provides strong evidence that the genetic determinants of adiponectin levels are shared with T2D using a Mendelian randomisation approach [13].

There have been numerous studies investigating the association of adiponectin gene and related polymorphisms with T2D. Two recent large meta-analyses showed that the $\mathrm{G}$ vs $\mathrm{C}$ allele of rs266729 might be a risk factor for T2D [42,43], while the rs17300539 A allele was shown to be a risk factor only in European Caucasians [43]. The latter SNP is in complete LD with rs16861209 that we have identified in our Caucasian populations.

The limitations of this study include the use of a single baseline serum adiponectin measurement which may not fully characterise long-term adiponectin exposure and effects. Nevertheless, other prospective studies 
that analysed repeated serum adiponectin measurements, on average one year apart, reported high intraclass correlations above $0.71[35,44]$, indicating high reproducibility of serum adiponectin levels. In the present study, total serum adiponectin levels were measured, whereas recent evidence suggests that the high molecular-weight form of adiponectin, rather than the low molecular-weight forms, may be the bioactive protein [45].

\section{Conclusions}

In summary, we have comprehensively analysed the genetic variants and haplotypes of $A D I P O Q$ and its two receptor genes using a tagSNP approach in two general populations and a diabetic cohort, in total comprising 3,322 well phenotyped subjects. We found that age, gender and BMI accounted for approximately one-third of the variation in serum adiponectin in the general population cohorts and just over one-fifth in a diabetic population. Variants in the $A D I P O Q$ gene accounted for $<5 \%$ of the variation in serum adiponectin and genetic variants in $A D I P O Q$ and its receptors does not appear to contribute to the risk of insulin resistance or metabolic syndrome. However, a multi-SNP genotypic risk score for ADIPOQ did associate with T2D independent of adiponectin levels. The factors that underlie the majority of the variation in serum adiponectin remain unknown, but given the potential beneficial effects of this adipokine, merit further study.

\section{Additional files}

Additional file 1: Details of the SNPs genotyped in this study. The location and minor allele frequency (from HapMap) of each tSNP are given, together with the allele frequency and genotype distribution in each cohort.

Additional file 2: Association of $A D I P O Q$ SNPs with serum adiponectin for each cohort. The marginal geometric mean values of adiponectin according to genotype at significant tSNPs are shown by individual cohort. Data are estimated marginal mean (MM) serum adiponectin $\mathrm{mg} / \mathrm{L}(95 \% \mathrm{Cl})$ after adjusting for age, gender and BMI. Pvalues were taken from the likelihood ratio test: model without genetic covariates vs model with genetic covariates. Q-values were calculated in each cohort using all 21 ADIPOQ tSNPs. ${ }^{*},+, \neq$ represents tSNPs that are in $L D$ in our study.

Additional file 3: Details of the additional SNPs captured by our tagSNP approach. Haploview was used to determine additional SNPS that were captured by the tSNPS genotyped in the present study $\left(r^{2} \geq 0.80, M A F>0.01\right)$. This analysis was not applicable to tSNPs $r 860291$, rs822395 and rs2241766 as they are not listed in HapMap. * tSNP associated with serum adiponectin in our cohort.

\section{Abbreviations}

MetS: Metabolic syndrome; T2D: Type 2 diabetes; GWAS: Genome-wide association study; SNP: Single nucleotide polymorphism; tSNP: Tag single nucleotide polymorphism; BHS: Busselton Population Health Survey; CUDAS: Carotid Ultrasound Disease Assessment Study; FDS: Fremantle Diabetes Study; IR: Insulin resistance; InHOMA-IR: Homeostatic model of assessment; BMI: Body mass index; NCEP: National Cholesterol Education Program; HWE: Hardy-Weinberg equilibrium; LD: Linkage disequilibrium.

\section{Competing interests}

The authors declare that they have no competing interests.

\section{Authors' contributions}

KEP drafted the manuscript and assisted in the collection, analysis, and interpretation of the data. GC, NMW, and SW carried out the analysis and interpretation of the data. JB and $\mathrm{JH}$ coordinated the data collection and interpretation of results. WD was responsible for the FDS data collection and validation. TD is the principal investigator of the FDS. All authors participated in the study design and coordination. All authors read and contributed to the final version of the manuscript.

\section{Acknowledgements}

Steven Wiltshire died prior to the publication of this paper. The co-authors would like to gratefully acknowledge his invaluable contribution to the inception and design of this study and to the analysis of results. The authors thank the Busselton Population Medical Research Foundation for access to the Busselton Health Survey data and the Busselton community for their long-standing cooperation and support for the Busselton Health Study. Special thanks also to the participants of Carotid Ultrasound Disease Assessment Study and Fremantle Diabetes Study, as well as the staff involved in the original data collection for each of these cohorts. We also thank the staff at the Western Australian DNA Bank for storage and preparation of the DNA used in the study. The current study was funded by a National Health and Medical Research Council Project Grant (NHMRC 513778). The Carotid Ultrasound Disease Assessment Study was supported by a grant-in-aid from the National Heart Foundation (GP97P 5002). The Fremantle Diabetes Study was supported by the Raine Foundation, University of Western Australia. TMED is supported by an NHMRC Practitioner Fellowship.

\section{Author details}

'School of Medicine and Pharmacology, Fremantle Hospital Unit, The University of Western Australia, Nedlands, Western Australia, Australia. ${ }^{2}$ Department of Diagnostic Molecular Genomics, PathWest, Queen Elizabeth II Medical Centre, Nedlands, Nedlands, Western Australia, Australia. ${ }^{3}$ School of Pathology and Laboratory Medicine, The University of Western Australia, Nedlands, Western Australia, Australia. ${ }^{4}$ Centre for Genetic Origins of Health and Disease, University of Western, Nedlands, Western Australia, Australia. ${ }^{5}$ School of Women's and Infants' Health, University of Western Australia, Nedlands, Western Australia, Australia. ${ }^{6}$ Centre for Medical Research, Western Australian Institute for Medical Research, The University of Western Australia, Nedlands, Australia. ${ }^{7}$ School of Population Health, The University of Western Australia, Nedlands, Western Australia, Australia. ${ }^{8}$ School of Medicine and Pharmacology, Sir Charles Gairdner Hospital Unit, University of Western Australia, Nedlands, Western Australia, Australia. ${ }^{9}$ Genetic Epidemiology and Biostatistics Platform, Ontario institute for Cancer Research, Toronto, Canada.

Received: 12 October 2012 Accepted: 17 January 2013

Published: 25 January 2013

\section{References}

1. Kadowaki T, Yamauchi T, Kubota N, Hara K, Ueki K, Tobe K: Adiponectin and adiponectin receptors in insulin resistance, diabetes, and the metabolic syndrome. J Clin Invest 2006, 116(7):1784-1792.

2. Jee SH, Sull JW, Lee JE, Shin C, Park J, Kimm H, Cho EY, Shin ES, Yun JE, Park JW, et al: Adiponectin concentrations: a genome-wide association study. Am J Hum Genet 2010, 87(4):545-552.

3. Heid IM, Henneman P, Hicks A, Coassin S, Winkler T, Aulchenko YS, Fuchsberger C, Song K, Hivert M-F, Waterworth DM, et al: Clear detection of ADIPOQ locus as the major gene for plasma adiponectin: Results of genome-wide association analyses including 4659 European individuals. Atherosclerosis 2010, 208(2):412-420.

4. Ling $H$, Waterworth DM, Stirnadel HA, Pollin TI, Barter PJ, Kesaniemi YA, Mahley RW, McPherson R, Waeber G, Bersot TP, et al: Genome-wide linkage and association analyses to identify genes influencing adiponectin levels: the GEMS Study. Obesity (Silver Spring) 2009, 17(4):737-744.

5. Richards JB, Waterworth D, O'Rahilly S, Hivert M-F, Loos RJF, Perry JRB, Tanaka T, Timpson NJ, Semple RK, Soranzo N, et al: A genome-wide association study reveals variants in $A R L 15$ that influence adiponectin levels. PLoS Genet 2009, 5(12):e1000768. 
6. Vasseur F, Helbecque N, Dina C, Lobbens S, Delannoy V, Gaget S, Boutin P, Vaxillaire $M$, Lepretre F, Dupont S, et al: Single-nucleotide polymorphism haplotypes in the both proximal promoter and exon 3 of the APM1 gene modulate adipocyte-secreted adiponectin hormone levels and contribute to the genetic risk for type 2 diabetes in French Caucasians. Hum Mol Genet 2002, 11(21):2607-2614.

7. Heid IM, Wagner SA, Gohlke H, Iglseder B, Mueller JC, Cip P, Ladurner G, Reiter R, Stadlmayr A, Mackevics V, et al: Genetic architecture of the APM1 gene and its influence on adiponectin plasma levels and parameters of the metabolic syndrome in 1,727 healthy Caucasians. Diabetes 2006, 55(2):375-384

8. Hivert M-F, Manning AK, MCAteer JB, Florez JC, Dupuis J, Fox CS, O'Donnell CJ, Cupples LA, Meigs JB: Common variants in the adiponectin gene (ADIPOQ) associated with plasma adiponectin levels, type 2 diabetes, and diabetes-related quantitative traits. Diabetes 2008, 57(12):3353-3359.

9. Menzaghi C, Trischitta V, Doria A: Genetic influences of adiponectin on insulin resistance, type 2 diabetes, and cardiovascular disease. Diabetes 2007, 56(5):1198-1209.

10. Henneman P, Aulchenko YS, Frants RR, Zorkoltseva IV, Zillikens MC, Frolich M, Oostra BA, van Dijk KW, van Duijn CM: Genetic architecture of plasma adiponectin overlaps with the genetics of metabolic syndrome-related traits. Diabetes Care 2010, 33(4):908-913.

11. Vasseur F, Meyre D, Froguel P: Adiponectin, type 2 diabetes and the metabolic syndrome: lessons from human genetic studies. Expert Rev Mol Med 2006, 8(27):1-12.

12. Mackevics V, Heid IM, Wagner SA, Cip P, Doppelmayr H, Lejnieks A, Gohlke H, Ladurner $G$, Illig T, Iglseder B, et al: The adiponectin gene is associated with adiponectin levels but not with characteristics of the insulin resistance syndrome in healthy Caucasians. Eur J Hum Genet 2006, 14(3):349-356.

13. Smith GD, Ebrahim S: 'Mendelian randomization': can genetic epidemiology contribute to understanding environmental determinants of disease? Int J Epidemiol 2003, 32(1):1-22.

14. Stefan N, Machicao F, Staiger H, Machann J, Schick F, Tschritter O, Spieth C, Weigert C, Fritsche A, Stumvoll M, et al: Polymorphisms in the gene encoding adiponectin receptor 1 are associated with insulin resistance and high liver fat. Diabetologia 2005, 48(11):2282-2291.

15. Crimmins NA, Martin LJ: Polymorphisms in adiponectin receptor genes ADIPOR1 and ADIPOR2 and insulin resistance. Obes Rev 2007, 8(5):419-423.

16. Kim JT, Kim Y, Cho YM, Koo BK, Lee EK, Shin HD, Jang HC, Choi JW, Oh B, Park KS: Polymorphisms of ADIPOR1 and ADIPOR2 are associated with phenotypes of type 2 diabetes in Koreans. Clin Endocrinol (Oxf) 2009, 70(1):66-74.

17. Hara K, Horikoshi M, Kitazato H, Yamauchi T, Ito C, Noda M, Ohashi J, Froguel $P$, Tokunaga $K$, Nagai $R$, et al: Absence of an association between the polymorphisms in the genes encoding adiponectin receptors and type 2 diabetes. Diabetologia 2005, 48(7):1307-1314.

18. Collins SC, Luan J, Thompson AJ, Daly A, Semple RK, O'Rahilly S, Wareham $\mathrm{NJ}$, Barroso I: Adiponectin receptor genes: mutation screening in syndromes of insulin resistance and association studies for type 2 diabetes and metabolic traits in UK populations. Diabetologia 2007, 50(3):555-562

19. Knuiman MW, Hung J, Divitini ML, Davis TM, Beilby JP: Utility of the metabolic syndrome and its components in the prediction of incident cardiovascular disease: a prospective cohort study. Eur J Cardiovasc Prev Rehabil 2009, 16(2):235-241.

20. Hung J, McQuillan BM, Thompson PL, Beilby JP: Circulating adiponectin levels associate with inflammatory markers, insulin resistance and metabolic syndrome independent of obesity. Int J Obes 2008, 32(5):772-779.

21. Davis TM, Bruce DG, Davis WA: Cohort profile: the Fremantle diabetes study. Int J Epidemiol 2012, doi:10.1093/ije/dys065.

22. Knuiman MW, Jamrozik K, Welborn TA, Bulsara MK, Divitini ML, Whittall DE: Age and secular trends in risk factors for cardiovascular disease in Busselton. Aust J Public Health 1995, 19(4):375-382.

23. Levy JC, Matthews DR, Hermans MP: Correct homeostasis model assessment (HOMA) evaluation uses the computer program. Diabetes Care 1998, 21(12):2191-2192.

24. Grundy SM, Cleeman JI, Daniels SR, Donato KA, Eckel RH, Franklin BA, Gordon DJ, Krauss RM, Savage PJ, Smith SC Jr, et al: Diagnosis and management of the metabolic syndrome: an american heart association/national heart, lung, and blood institute scientific statement: executive summary. Crit Pathw Cardiol 2005, 4(4):198-203.
25. Carter KW, McCaskie PA, Palmer LJ: SimHap GUl: an intuitive graphical user interface for genetic association analysis. BMC Bioinforma 2008, 9:557.

26. Benjamini $Y$, Hochberg $Y$ : Controlling the false discovery rate: a practical and powerful approach to multiple testing. J R Stat Soc B 1995, 85:289-300

27. Barrett JC, Fry B, Maller J, Daly MJ: Haploview: analysis and visualization of LD and haplotype maps. Bioinformatics 2005, 21(2):263-265.

28. Gauderman WJ: Sample size requirements for association studies of gene-gene interaction. Am J Epidemiol 2002, 155(5):478-484.

29. Siitonen N, Pulkkinen L, Lindstrom J, Kolehmainen M, Eriksson JG, Venojarvi M, Ilanne-Parikka P, Keinanen-Kiukaanniemi S, Tuomilehto J, Uusitupa M: Association of ADIPOQ gene variants with body weight, type 2 diabetes and serum adiponectin concentrations: the Finnish Diabetes Prevention Study. BMC Med Genet 2011, 12:5

30. Mather KJ, Christophi CA, Jablonski KA, Knowler WC, Goldberg RB, Kahn SE, Spector T, Dastani Z, Waterworth D, Richards JB, et al: Common variants in genes encoding adiponectin (ADIPOQ) and its receptors (ADIPOR1/2), adiponectin concentrations, and diabetes incidence in the Diabetes Prevention Program. Diabet Med 2012, 29(12):1579-1588.

31. Dastani Z, Hivert MF, Timpson N, Perry JR, Yuan X, Scott RA, Henneman P, Heid IM, Kizer JR, Lyytikainen LP, et al: Novel loci for adiponectin levels and their influence on type 2 diabetes and metabolic traits: a multiethnic meta-analysis of 45,891 individuals. PLoS Genet 2012, 8(3):e1002607

32. Croteau-Chonka DC, Wu Y, Li Y, Fogarty MP, Lange LA, Kuzawa CW, McDade TW, Borja JB, Luo J, AbdelBaky O, et al: Population-specific coding variant underlies genome-wide association with adiponectin level. Hum $\mathrm{Mol}$ Genet 2012, 21(2):463-471.

33. Warren LL, Li L, Nelson MR, Ehm MG, Shen J, Fraser DJ, Aponte JL, Nangle $\mathrm{KL}$, Slater AJ, Woollard PM, et al: Deep resequencing unveils genetic architecture of ADIPOQ and identifies a novel low-frequency variant strongly associated with adiponectin variation. Diabetes 2012, 61(5):1297-1301.

34. Dickson SP, Wang K, Krantz I, Hakonarson H, Goldstein DB: Rare variants create synthetic genome-wide associations. PLOS Biol 2010, 8(1):e1000294.

35. Dhillon PK, Penney KL, Schumacher F, Rider JR, Sesso HD, Pollak M, Fiorentino M, Finn S, Loda M, Rifai N, et al: Common polymorphisms in the adiponectin and its receptor genes, adiponectin levels and the risk of prostate cancer. Cancer Epidemiol Biomarkers Prev 2011, 20(12):2618-2627.

36. Cohen SS, Gammon MD, North KE, Millikan RC, Lange EM, Williams SM, Zheng W, Cai Q, Long J, Smith JR, et al: ADIPOQ, ADIPOR1, and ADIPOR2 polymorphisms in relation to serum adiponectin levels and BMI in black and white women. Obesity (Silver Spring) 2011, 19(10):2053-2062.

37. Halvatsiotis I, Tsiotra PC, Ikonomidis I, Kollias A, Mitrou P, Maratou E, Boutati E, Lekakis J, Dimitriadis G, Economopoulos T, et al: Genetic variation in the adiponectin receptor 2 (ADIPOR2) gene is associated with coronary artery disease and increased ADIPOR2 expression in peripheral monocytes. Cardiovasc Diabetol 2010, 9:10.

38. Chung CM, Lin TH, Chen JW, Leu HB, Yang HC, Ho HY, Ting CT, Sheu SH, Tsai WC, Chen JH, et al: A genome-wide association study reveals a quantitative trait locus of adiponectin on $\mathrm{CDH} 13$ that predicts cardiometabolic outcomes. Diabetes 2011, 60(9):2417-2423.

39. Morisaki H, Yamanaka I, Iwai N, Miyamoto Y, Kokubo Y, Okamura T, Okayama A, Morisaki T: CDH13 gene coding T-cadherin influences variations in plasma adiponectin levels in the Japanese population. Hum Mutat 2012, 33(2):402-410.

40. Wu Y, Li Y, Lange EM, Croteau-Chonka DC, Kuzawa CW, McDade TW, Qin L, Curocichin G, Borja JB, Lange LA, et al: Genome-wide association study for adiponectin levels in Filipino women identifies $\mathrm{CDH} 13$ and a novel uncommon haplotype at KNG1-ADIPOQ. Hum Mol Genet 2010, 19(24):4955-4964.

41. Bowden DW, An SS, Palmer ND, Brown WM, Norris JM, Haffner SM, Hawkins GA, Guo X, Rotter JI, Chen YD, et al: Molecular basis of a linkage peak: exome sequencing and family-based analysis identify a rare genetic variant in the ADIPOQ gene in the IRAS Family Study. Hum Mol Genet 2010, 19(20):4112-4120.

42. Han LY, Wu QH, Jiao ML, Hao YH, Liang LB, Gao LJ, Legge DG, Quan H, Zhao MM, Ning $N$, et al: Associations between single-nucleotide polymorphisms (+45 T>G, $+276 \mathrm{G}>\mathrm{T},-11377 \mathrm{C}>\mathrm{G},-11391 \mathrm{G}>\mathrm{A}$ ) of adiponectin gene and type 2 diabetes mellitus: a systematic review and meta-analysis. Diabetologia 2011, 54(9):2303-2314. 
43. Gong M, Long J, Liu Q, Deng HC: Association of the ADIPOQ rs17360539 and rs266729 polymorphisms with type 2 diabetes: a meta-analysis. Mol Cell Endocrinol 2010, 325(1-2):78-83.

44. Pischon T, Hotamisligil GS, Rimm EB: Adiponectin: stability in plasma over 36 hours and within-person variation over 1 year. Clin Chem 2003, 49(4):650-652

45. Pajvani UB, Hawkins M, Combs TP, Rajala MW, Doebber T, Berger JP, Wagner JA, Wu M, Knopps A, Xiang AH, et al: Complex distribution, not absolute amount of adiponectin, correlates with thiazolidinedione-mediated improvement in insulin sensitivity. J Biol Chem 2004, 279(13):12152-12162.

\section{doi:10.1186/1471-2350-14-15}

Cite this article as: Peters et al:: A comprehensive investigation of variants in genes encoding adiponectin (ADIPOQ) and its receptors (ADIPOR1/R2), and their association with serum adiponectin, type 2 diabetes, insulin resistance and the metabolic syndrome. BMC Medical Genetics 2013 14:15.

\section{Submit your next manuscript to BioMed Central and take full advantage of:}

- Convenient online submission

- Thorough peer review

- No space constraints or color figure charges

- Immediate publication on acceptance

- Inclusion in PubMed, CAS, Scopus and Google Scholar

- Research which is freely available for redistribution 